\title{
OPIO Y COLONIALISMO: REFLEXIONES SOBRE EL PAPEL DEL OPIO EN LA PENETRACIÓN COLONIAL EUROPEA EN ASIA Y CHINA
}

\author{
JOSÉ ANTONIO CANTÓN ÁLVAREZ \\ Universidad de Granada
}

\section{Introducción}

El comercio y el consumo de opio en Asia, y particularmente en China, durante los siglos XVIII y XIX, así como sus implicaciones en la historia contemporánea de dichas regiones, han sido objeto de un debate que se ha revitalizado durante los últimos veinte años. ${ }^{1}$ No obstante, han sido pocos los historiadores que han tratado esta temática a través del enfoque de la historia global, como parte de la expansión colonial europea en Asia, y lo han hecho centrándose en las Guerras del Opio. ${ }^{2}$ Este artículo pretende seguir la estela de esta nueva corriente de

Este artículo fue recibido por la dirección de la revista el 2 de diciembre de 2014 y aceptado para su publicación el 15 de enero de 2015.

${ }^{1}$ Véase John Y. Wong, Deadly Dreams: Opium and the Arrow War (1856-1860) in China, Cambridge, Cambridge University Press, 1998; Timothy Brook y Bob T. Wakabayashi (eds.), Opium Regimes. China, Britain and Japan, 1839-1952, BerkeleyLos Ángeles, University of California Press, 2000; Frank Dikötter, Lars Laamann y Zhou Xun, Narcotic Culture: A History of Drugs in China, Chicago, University of Chicago Press, 2004, y Zheng Yangwen, The Social Life of Opium in China, Cambridge, Cambridge University Press, 2005.

${ }^{2}$ V'éase Giovanni Arrighi, Iftikhar Ahmad y Miin-wen Shih, "Western Hegemonies in World Historical Perspective", en Giovanni Arrighi, Chaos and Governance in the Modern World System, Minneapolis, University of Minnesota Press, 1999, pp. 227236; Carl A. Trocki, Opium, Empire and the Global Political Economy, Londres, Routledge, 1999, passim, y Hans Derk, History of the Opium Problem: The Assault on the East, ca. 1600-1950, Leiden, Brill, 2012, pp. 133-380. El historiador chino Zhong Weimin ha planteado las limitaciones de este enfoque: Zhong Weimin 仲伟民, Chaye yu yapian: shijiu shiji quanqiubua zhong de Zhongguo 茶叶与鸦片: 十九世纪经济全球 化中的中国, Beijing, Shenghuo, Dushu, Xinzhi Sanlian Shudian 生活・读书・新知三 联书店, 2010, pp. 28-33. 
estudio, al plantear que el comercio del opio inglés en China durante los siglos XVIII y XIX no surgió como una actividad ex novo, sino que se asentó sobre dinámicas económicas regionales muy amplias, que echan raíces en procesos sólo entendibles desde una óptica longue durée. En este sentido, debe ser entendido como el punto culminante de un largo proceso, iniciado siglos atrás, de comprensión de las redes mercantiles a ambos lados del Índico, que tuvo sus bases en la experimentación de los portugueses y los holandeses con el comercio del opio en sus actividades productivas. Sólo tras siglos de exploración se consiguió entender su impresionante potencial, como mercancía capaz de funcionar como un elemento disruptivo de las relaciones comerciales. Estas experiencias permitieron al Imperio británico encontrar la "herramienta" que necesitaba para despolarizar un entorno económico -el de los mares de China y el Sureste Asiático- caracterizado por el papel central del Imperio Qing en los intercambios comerciales, gracias a los cambios producidos en el consumo de opio durante el siglo XVII, lo que provocó la introducción del tabaco en Asia. En este proceso, se crearon nuevas relaciones coloniales en la periferia y se rompió la capacidad del imperio para gestionar su propia economía. Sólo cuando dicho cambio se hubo consumado, la superioridad naval y militar europea apareció como vía para evitar cualquier intento de reversión de dicha transformación. En atención a estos aspectos, el objetivo de estas líneas es replantear, desde una óptica global y a luz de las nuevas aportaciones en el campo, el papel del mercado británico de opio en China durante los siglos XVIII y XIX, así como su vinculación con la violenta entrada del entonces imperio manchú Qing en la esfera colonial. En este sentido, mi intención es contextualizar el comercio del opio dentro de la expansión colonial europea en el sur, este y sureste de Asia y explicar, a su vez, su utilización como herramienta durante la apertura colonial de China.

El estudio de la historia del opio, como el de cualquier otra droga, ${ }^{3}$ es una tarea que requiere de un enfoque interdisciplinario, especialmente si tenemos en cuenta que es uno de los que

${ }^{3}$ En pos de una redacción más fluida, usaré el término drogas para referirme estrictamente a las sustancias psicoactivas. 
más intrusiones de otras disciplinas ha recibido, debido a las implicaciones morales y políticas que su producción, comercio y consumo tienen en el mundo actual. Diversos investigadores han planteado que estas últimas son fruto de la creación de un discurso moralizante en nuestra concepción de su historia, cuyo origen aún es discutido ${ }^{4}$ y que ha pesado no sólo en la percepción de la academia occidental, sino igualmente en la china. ${ }^{5}$ Este problema hace difícil una visión global de la cuestión del opio dentro de un ámbito mucho más amplio, como es la transformación de la relación del ser humano con la naturaleza a raíz de las progresivas revoluciones agrícolas, y en concreto con aquellos productos fungibles con cierta capacidad de generar un hábito de consumo o adicción, lo cual está a su vez relacionado con el surgimiento del capitalismo moderno.

A diferencia de otras mercancías, como el algodón o las especias, cuyo comercio es esencial en nuestra comprensión de la formación del capitalismo moderno, las drogas se distinguen por ser sustancias que, por sus propiedades psicoactivas, juegan un papel muy complejo en las culturas de consumo en las que se desenvuelven, especialmente por su función dentro de la gestión psicológica del trabajo y el reposo, en lo que algunos especialistas han dado en llamar la "gestión química de la vida cotidiana". ${ }^{6}$ En lo que a esta investigación se refiere, este factor plantea aspectos esenciales para comprender el papel que juega el consumo de determinadas drogas en la organización del trabajo y en la reorganización de los códigos sociales cuando su consumo se ve transformado por elementos externos a un colectivo o, incluso, cuando son introducidas en un contexto social al que son ajenas. A la vista de la escasa investigación

${ }^{4}$ A pesar de la importancia que la cuestión moralizante tiene para el asunto que investigamos, su complejidad hace imposible tratarla en este artículo. Sobre el tema véase Antonio Escohotado, Historia general de las drogas, Madrid, Espasa, 2005, pp. 258 259; Derk, History of the Opium Problem, op. cit., pp. 3-33; Louise Foxcroft, The Making of Addiction: The "Use and Abuse" of Opium in Nineteenth-Century, Aldershot, Ashgate Publishing, 2013, pp. 62 y ss., y Geoffrey Hunt y Judith C. Barker, "Socio-cultural Anthropology and Alcohol and Drug Research: Towards a Unified Theory”, Social Science EMedicine, vol. 53, núm. 2, 2001, pp. 165-188.

${ }^{5}$ Brook y Wakabayashi (eds.), Opium Regimes, op. cit., p. 2.

${ }^{6}$ William R. Miller y Kathleen M. Carroll, Rethinking Substance Abuse: What the Science Shows, and What We Should Do about It, Nueva York, Guilford Press, 2006, p. 208. 
desarrollada en torno de esta cuestión, Drugs, Labor and Colonial Expansion, obra colectiva dirigida por Daniel Bradburd y William Jankowiak, propone algunas de las líneas argumentales más útiles para la constitución del marco teórico de este estudio. Así, resulta esclarecedora su elegante apropiación de la ruptura de los principios de intercambiabilidad de Appudurai ${ }^{7}$ en el contexto de la introducción de una droga, como mercancía, en realidades sociales a las que es ajena. Esto nos ayuda a ver las drogas como "bienes que, por su naturaleza, crean y mantienen intercambios y contextos donde "valor y precio han quedado [...] desconectados"”. ${ }^{8}$

Así, la capacidad de las drogas para forzar intercambios sin ninguna posibilidad de equivalencia les confiere un gran poder para trastocar los colectivos sociales con los que tienen contacto, siempre que éstos no tengan conocimiento de ellas. Al partir de este potencial, Bradburd y Jankowiak plantean un modelo en el que el papel de las drogas dentro del proceso colonial se ajusta a dos fases. Una primera basada en la "seducción", en la que se induce a la gente a proveer bienes o trabajo, y una segunda en la que las drogas son empleadas para aumentar la intensidad o la duración del trabajo, una vez que está controlado. Así, la primera tiene relación directa con la "presencia" europea en distintas partes del globo como paso previo a la segunda, relacionada con el "control" de las drogas. ${ }^{9}$ Esta lúcida distinción que hacen Brardburd y Jankowiak es, como veremos, muy útil para nuestra aproximación a la cuestión, en tanto puede argumentarse que los europeos hicieron uso del comercio de diversas drogas cuando no encontraron el modo de controlar con quién o bajo qué circunstancias los nativos comerciaban. ${ }^{10}$ La idea de esta característica de las drogas como

7 Arjun Appadurai "Introduction: Commodities and the Politics of Value", en Arjun Appadurai (ed.), The Social Life of Things: Commodities in Cultural Perspective, Cambridge, Cambridge University Press, 1986, p. 14.

8 " [...] goods that, by their nature, create and maintain exchanges or contexts where 'value and price have become [...] completely unyoked' outside situations of extreme hardship". Daniel Bradburd y William Jankowiak, "Drugs, Desire, and European Economic Expansion", en Daniel Bradburd y William Jankowiak (eds.), Drugs, Labor and Colonial Expansion, Tucson, The University of Arizona Press, 2003, p. 9.

${ }^{9}$ Ibid., pp. 12-13.

${ }^{10}$ Ibid., p. 20. 
posibles herramientas de control comercial da un nuevo significado al comercio del opio en Asia durante los siglos XVIII y XIX, así como a su papel en la empresa colonial europea en dicho continente.

\section{El opio y los inicios del colonialismo en Asia}

Para contextualizar en su verdadera dimensión el tema del opio en el escenario europeo y asiático en los últimos tres siglos es necesario hablar de esta sustancia con anterioridad, a fin de presentar su historia como la de un viaje de ida y vuelta entre ambos continentes. A pesar de que la relación del ser humano con el opio puede rastrearse hasta la época prehistórica, su consumo ha sido esencialmente vinculado al continente asiático en una tradición que se refleja en los estudios anglosajones sobre la cuestión publicados en la primera mitad del siglo Xx, como es el caso del historiador estadounidense David Edward Owen, quien definía el opio como un "vicio distintivo de Oriente". ${ }^{11}$ En cambio, estudios arqueológicos apuntan a que el origen del consumo de la adormidera (Papaver somniferum) pudo darse en el sur de la península ibérica; ${ }^{12}$ también se han encontrado muestras de su uso - datadas en fechas del 3000 al 2500 a.n.e.- en yacimientos de Alemania, Italia y Suiza. ${ }^{13}$ Del mismo modo, ha sido ampliamente estudiada su mención en las obras de diversos nichos civilizatorios, como Oriente Medio o Egipto; así como la función desempeñada por el opio en las culturas egipcia y griega, o su "fetichización" en la medicina de esta última como base para la confección de la triaca

${ }^{11}$ David E. Owen, British Opium Policy in China and India, New Haven, Yale University Press, 1934, p. 2; Escohotado, Historia general de las drogas, op. cit., pp. 171, 258259; Alan Baumler (ed.), Modern China and Opium: A Reader, Ann Arbor, University of Michigan Press, 2001, pp. 28-34.

${ }^{12}$ Más concretamente en la Cueva de los Murciélagos, en el municipio de Zuheros, provincia de Córdoba. Andrew Sherratt, "Sacred and Profane Substances: The Ritual Use of Narcotics in Later Neolithic Europe", en P. Garwood, D. Jennings, R. Skeates y J. Toms (eds.), Sacred and Profane: Proceedings of a Conference on Archaeology, Ritual and Religion, Oxford, University Committee for Archaeology Monographs, 1991, p. 62.

${ }^{13}$ Mark D. Merlin, On the Trail of the Ancient Opium Poppy, Nueva Jersey, Fairleigh Dickinson University Press, 1984, pp. 135-145. 
$(\theta \eta \rho \iota \alpha \kappa \eta) .{ }^{14}$ Ya en la época medieval, esta tradición sirvió a los árabes como medio para tomar conciencia de las propiedades de la droga, debido a la adopción de la tradición galénica y triacal por Ibn Sinna (Avicena, 980-1037), ${ }^{15}$ y sería a través de la primera expansión del islam como llegaría a otras regiones del continente asiático, como China, Tailandia o India; ${ }^{16}$ de lo que se desprende que el opio fue quizás la primera droga en verse afectada por las etapas más tempranas de la globalización. De este modo, ya antes de 1498, el látex de la adormidera era una mercancía que había sido objeto de un incipiente comercio en distintas regiones del continente asiático, si bien la parquedad de las fuentes que lo refieren, $\mathrm{da}$ a atender que su volumen era ciertamente limitado y que se utilizaba por sus propiedades medicinales. ${ }^{17}$ No obstante, esto no pareció impedir que la relación entre opio y colonialismo europeo en Asia comenzase en los mismos

${ }^{14}$ Susan P. Mattern, The Prince of Medicine: Galen in the Roman Empire, Oxford, Oxford University Press, 2013, pp. 217-218; John Scarborough, "The Opium Poppy in Hellenistic and Roman Medicine", en Roy Porter (ed.), Drugs and Narcotics in History, Cambridge, Cambridge University Press, 1997, pp. 17-18; Escohotado, Historia general de la drogas, op. cit., pp. 143-144.

${ }^{15}$ El papel del opio en la práctica médica del mundo musulmán sigue siendo lamentablemente un apartado que no ha recibido un estudio exhaustivo. Quizás el más importante realizado hasta la fecha sea la aproximación de Tilbi a su papel en la farmacología del califato Abbasí. Selma Tilbi, The Medicinal Use of Opium in NinthCentury Baghdad, Leiden, Brill, 2006, pp. 170-183.

${ }^{16}$ Las referencias más antiguas al opio en la cultura china son las aparecidas en Huibui Yaofang 回回药方 (Recetario musulmán), obra colectiva escrita en el siglo XIII. Véase Gong Yingyan 龚缗晏, Yapian de chuanbo yu duibua yapian maoyi 鸦片的传 播与对华鸦片贸易, Beijing, Dongfang Chubanshe 东方出版社, 1999, pp. 58-71, y Angela Schottenhammer, "Huihui Medicine and Medicinal Drugs in Yuan China", en Morris Rossabi (ed.), Eurasian Influences on Yuan China, Singapur, Institute of Southeast Asian Studies, 2013, pp. 75-102. Por su parte, en el reino de Ayutthaya (Tailandia) el consumo de opio se convirtió en una preocupación del recién ascendido rey Ramathibodi I, quien en 1360 hizo incluir en su código legislativo una ley que lo prohibiese. Kasian Tejapira, "Pigtail: A Prehistory of Chineseness in Siam”, en Tong Chee Kiong y Chan Kwok B. (eds.), Alternate Identities: The Chinese of Contemporary Thailand, Leiden, Brill, 2001, pp. 54-55. El caso de la entrada del opio en India es, no obstante, más difícil de datar con exactitud, aunque los estudios más clásicos la ubican en el siglo Ix: R. N. Chopra, Indigenous Drugs of India, Calcuta, The Art Press, 1933, pp. 205-206.

${ }_{17}$ A falta de fuentes que nos den información más detallada en otros entornos geográficos, la prohibición del consumo de opio en Tailandia, en 1360, o las expediciones mandadas, en 1483, por el emperador Chenghua (1447-1487) dan cuenta de la amplitud de este mercado precolonial en torno del látex de la adormidera. Zheng, The Social Life of Opium in China, op. cit., pp. 10-15. 
orígenes de este último. Esto es lo que muestra la carta enviada por Alfonso de Albuquerque al rey Manuel I de Portugal, el 1 de diciembre de 1513, en la que aconsejaba al monarca que diera orden de plantar adormidera en las islas Azores para aprovechar la ventaja estratégica que supondría el control de su comercio en India, sobre todo tras las incursiones realizadas por los lusos en el puerto de Adén ese mismo año. ${ }^{18}$ Aparte de ser la primera muestra documental europea de la presencia del opio como una mercancía de relativa importancia en las rutas comerciales en el siglo XVI, este documento tiene una relevancia sustancial por reflejar la intención del conquistador portugués de hacer de la adormidera un cash crop, extrapolando la incipiente mercantilización moderna del azúcar al opio, y de crear en las Azores una nueva "Madeira del opio" que alimentase el desabastecido gran consumo en India. ${ }^{19}$ Esta intención nunca llegó a materializarse, lo cual puede explicarse no sólo por la incapacidad de los lusos de tomar el puerto de Adén, lo que les hubiese permitido controlar la ruta comercial del mar Rojo, sino principalmente por el hecho de que en el subcontinente indio ya existía una amplia red productiva de adormidera, como lo demuestra la descripción del comercio del opio en Goa que dio el médico portugués García da Orta tras su llegada a India en $1538 .^{20}$ Así, se entiende que la alta valoración del opio egipcio en el mercado indio hizo que Albuquerque no reparase en el local, menos apreciado.

Además, el sueño de Albuquerque era inviable ya que, a diferencia del caso del azúcar, una mercantilización colonial del opio habría supuesto hacer lo mismo para un mercado externo

${ }^{18}$ Raymondo A. de Bulhão Pato (ed.), Cartas de Affonso de Albuquerque, seguidas de documentos que as elucidam, t. I, Lisboa, Typographia da Academia Real das Sciencias, 1884, p. 174.

${ }^{19}$ Sin duda, Alfonso de Albuquerque se vio motivado por la entonces boyante explotación de caña de azúcar afincada en dicha isla, la cual asistía a sus últimos años de prosperidad. Jason W. Moore, "Madeira, Sugar, and the Conquest of Nature in the 'First' Sixteenth Century, Part I: From 'Island of Timber' to Sugar Revolution, 1420-1506”, Review: A Journal of the Fernand Braudel Center, vol. XxxII, núm. 4, 2009, pp. 345-390; y Jason W. Moore, "Madeira, Sugar, and the Conquest of Nature in the 'First' Sixteenth Century, Part II: From Local Crisis to Commodity Frontier, 1506-1530”, Review: A Journal of the Fernand Braudel Center, vol. xxxiII, núm. 1, 2010, pp. 1-24.

${ }^{20}$ Garcia De Orta, Colóquios dos simples e drogas da India, vol. 2, Lisboa, Academia Real das Sciencias de Lisboa, 1891 (orig. 1563), p. 173. 
al reino de Portugal, de modo que el monopolio comercial era un requisito esencial para el éxito del proyecto. En tanto que esta última premisa nunca llegó a cumplirse, su incapacidad de hacer una mercantilización moderna del opio puede plantearse como una de las muchas pruebas que permite negar la supuesta centralidad de los portugueses como actores comerciales en el Índico entre los siglos XVI y XVIII. ${ }^{21}$ La crítica planteada por Das Gupta y Pearson demuestra que la dominancia de una supuesta "economía-mundo" no se daría sino hasta la conquista de Bengala por los británicos, en 1757, en un proceso en el que los europeos necesitaron coexistir antes de llevar a cabo un dominio efectivo a través de la reconversión de modelos comerciales ya existentes. ${ }^{22}$ En este sentido, la empresa portuguesa en Asia durante dicho periodo debe ser vista como un elemento mínimo dentro de un escenario comercial mucho más amplio y complejo.

\section{El opio en la ruptura del sistema comercial internacional chino}

Sin embargo, el comercio del opio y sus efectos se hicieron notar en el área de influencia comercial china mucho antes de que los británicos invadiesen India. Las fuentes occidentales no sólo hacen eco de su peso en India en el siglo XVI, como nos dio a entender Albuquerque, sino que además dan muestras

${ }^{21}$ Este planteamiento tiene sus raíces en la revisión historiográfica sobre la cuestión que tuvo lugar durante las décadas de 1980 y 1990; véase Ashin das Gupta y Michael N. Pearson, India and the Indian Ocean, 1500-1800, Oxford, Oxford University Press, 1987. Sanjay Subrahmanyam, The Political Economy of Commerce. Southern India, Cambridge, Cambridge University Press, 1990. Respecto a este particular, Frank explicó la creación de esta imagen como fruto del eurocentrismo reinante en la academia, y por la inexistencia o falta de estudios de documentación que no fuesen europeos y que escapasen a ese sesgo: Andre G. Frank, ReOrient: Global Economy in the Asian Age, Berkeley-Los Ángeles, University of California Press, 1998, pp. 20-34. Otros historiadores, como Klein, justifican la penetración de lusos y holandeses en este entorno por la inestabilidad política y económica de ciertas regiones durante el periodo. Peter Klein, "The China Seas and the World Economy between the Sixteenth and Nineteenth Centuries: The Changing Structure of World Trade”, en Carl-Ludwig Holtfrerich (ed.), Interaction in the World Economy. Perspectives from International Economic History, Londres, Harvester, 1989, pp. 86-87.

${ }^{22}$ Das Gupta y Pearson, India and the Indian Ocean, op. cit., p. 20. 
de que era una de las principales mercancías de un tráfico que se extendía a los principales puertos del entramado comercial dominado por los “juncos” chinos, y que unía los mares de China con la sección occidental del Indico, en el que el "País del Centro" era uno de los principales compradores de dicha droga. ${ }^{23}$ Las fuentes Ming hacen ver que esta preponderancia podría estar motivada no sólo por la centralidad del imperio en dicho sistema comercial, sino también por un cambio sustancial en la percepción de la droga en el País del Centro, en el que junto a su uso medicinal se dan las primeras muestras de su consumo recreativo en la corte imperial. ${ }^{24}$

Respecto a este particular, resulta interesante la perspectiva holística del historiador japonés Takeshi Hamashita, quien ubica un vasto sistema económico "sinocentrado" en las regiones del sureste, noreste, centro y noroeste del continente asiático, que operó hasta bien entrado el siglo XIX y que hacía uso del tributo como sistema en las relaciones económicas y mercantiles. ${ }^{25}$ Esta perspectiva nos permite ver la presencia europea en Asia, durante los siglos XVI y XVII, más como un proceso de "penetración" que como una conquista progresiva. Hasta la segunda mitad del siglo XIX, los europeos hubieron de realizar sus actividades comerciales dentro de este modelo, lo que explicaría, entre otras cosas, el trato que recibieron los comerciantes europeos, tanto en la época Ming como en la Qing, dentro del sistema comercial articulado a través de Cantón. En este sentido, y conforme el esquema de Bradburd y Jankowiak mencionado anteriormente, el opio sería una herramienta fundamental para la desestructuración del sistema sinocéntrico propuesto por Hamashita, primero en su periferia y después en su centro, como dos partes integradas de un mismo proceso.

${ }^{23}$ Duarte Barbosa, Livro em que se dá relação do que viu e oviu no oriente Duarte Barbosa, Lisboa, Divisão de publicações e biblioteca agência geral das colònias, 1956 (orig. 1516), pp. 201 y ss., y 218- 219.

${ }^{24} \mathrm{Si}$ bien este proceso se inicio a partir del año 1483, durante el reinado del emperador Chenghua, la época floreciente de este nuevo comercio fue la segunda mitad del siglo XVI y los inicios del siglo XVII, concretamente durante el reinado del emperador Wanli (1573-1620). Zheng, The Social Life of Opium in China, op. cit., pp. 10-24.

25 Takeshi Hamashita, China, East Asia and the Global Economy: Regional and Historical Perspectives, Oxon, Routledge, 2008, pp. 12-26. 
La historiografía más tradicional nos ha proporcionado una imagen de las relaciones comerciales chinas durante los Qing, basada en el sistema comercial de "puerto único" 一口通商, por el que todo el comercio internacional con China se articulaba a través de las trece agencias comerciales monopolísticas de Cantón $;^{26} \sin$ embargo, no corresponde a la configuración que tenía a la llegada de los manchúes al poder. Las primeras etapas del comercio del opio llevado a cabo por los europeos en el siglo XVIII se dieron en un periodo marcado por una intensa expansión comercial en el Sureste Asiático, que tiene sus orígenes, como se ha planteado, en 1680. Concretamente, dicha expansión comercial se reinició tras el hiato provocado por la invasión manchú y los conflictos que siguieron una vez que, en 1684, el emperador Kangxi abolió el cierre portuario 海禁 impuesto a causa de las luchas contra el pirata pro-Ming Coxinga, tras la conquista de Taiwan, en 1683. Dicha apertura vino a dar comienzo a un nuevo periodo en las relaciones comerciales chinas con el exterior, caracterizado por un impulso del libre comercio en la política económica imperial. ${ }^{27}$

$\mathrm{Al}$ partir de estas premisas entendemos que el estudio del comercio del opio en Asia, antes de 1757, requiere de un enfoque que integre los estudios sinológicos y los del Sudeste Asiático. El sistema de relaciones comerciales desarrollado entre ambas regiones, a partir del siglo XVII, funcionaba a través de redes de parentesco organizadas que permitieron un control de la diáspora que, aunque extraoficial, servía particularmente a los intereses económicos del imperio. ${ }^{28}$ Entre los destinos de esta gran emigración se encontraba la isla de Java, en aquel entonces

${ }^{26}$ John K. Fairbank, Trade and Diplomacy on the China Coast: The Opening of the Treaty Ports, 1842-1854, Cambridge, Harvard University Press, 1953. La cuestión ha recibido un estudio más pormenorizado y actualizado, en el que destacan dos ensayos de Paul A. Van Dyke: The Canton Trade: Life and Enterprise on the China Coast, 17001845, Hong Kong, Hong Kong University Press, 2005, y Merchants of Canton and Macao: Politics and Strategies in Eighteenth-Century Chinese Trade, Hong Kong, Hong Kong University Press, 2011.

${ }^{27} \mathrm{La}$ falta de estudios sobre dicho periodo ha comenzado a subsanarse recientemente. Gang Zhao, The Qing Opening to the Ocean: Chinese Maritime Policies, 16841757, Honolulú, Hawaii University Press, 2013, pp. 79-98.

${ }^{28}$ Kwee Hui Kian, "Pockets of Empire: Integrating the Studies on Social Organizations in Southeast China and Southeast Asia", Comparative Studies of South Asia, Africa and the Middle East, vol. 27, núm. 3, 2007, pp. 616-632. 
dominada por los holandeses, representados por la Vereenigde Oost-Indische Compagnie. Si bien en la historiografía tradicional se ha justificado la importancia de la empresa holandesa en el Sudeste Asiático por su fuerza disruptiva de los patrones comerciales asiáticos anteriores a las conquistas de Macassar en 1667, y la subsecuente caída en desgracia de puertos como Banten, ${ }^{29}$ estos efectos no pudieron evitar la creciente influencia de los comerciantes chinos en la región, la cual fue fuente de diversos conflictos. ${ }^{30}$

Asimismo, Java fue una de las primeras regiones tributarias de China en utilizar el opio como artículo, durante el siglo XV en la corte imperial china, junto con los reinos de Bengala y de Ayutthaya, cuyas ruinas se encuentran en la actual Tailandia. En esta época, esta droga constituía una pequeña parte del gran lote del mercado tributario con China, donde comenzó a consumirse por razones extramedicinales. Sin embargo, llegado el siglo XVII, el consumo de la droga en Indonesia experimentó importantes cambios a raíz de la llegada del tabaco a Asia, ${ }^{31}$ ya que ambas drogas comenzaron a ser consumidas a la vez en una mezcla conocida como madak. ${ }^{32}$ Este cambio favoreció la subsecuente extensión de la práctica a Malasia y al resto de los países bañados por el mar de China meridional, y otorgó una

${ }^{29}$ Véase de Niels Steensgaard, "The Companies as a Specific Institution in the History of European Expansion”, en L. Blussé y F. Gaastra (eds.), Companies and Trade: Essays on Overseas Trading Companies during the Ancient Regime, Leiden, Leiden University Press, 1981, pp. 245-264, y “The Dutch East India Company as an Institutional Innovation”, en M. Aymard (ed.), Dutch Capitalism and World Capitalism, Cambridge, Cambridge University Press, 1982, pp. 235-257.

${ }^{30}$ M. Ricklefs, "The Crisis of 1740-1 in Java: The Javanese, Chinese, Madurese and Dutch, and the Fall of the Court of Kartasura", Bijdragen tot de Taal-, Land-en Volkenkunde, vol. 139, núm. 2, 1983, pp. 268-290.

${ }^{31}$ Jordan Goodman, Tobacco in History: The Cultures of Dependence, Londres, Routledge, 2005, pp. 50-52, y Sander L. Gilman y Zhou Xun, "Introduction", en Sander L. Gilman y Zhou Xun (ed.), Smoke: A Global History of Smoking, Londres, Reaktion Books, 2004, pp. 9-28.

${ }^{32}$ El explorador inglés Edmund Scott documentó esta práctica en la ciudad de Bantam, en 1606. Edmund Scott, An Exact Discourse of the Subtilties, Fashishions [sic], Pollicies, Religion and Ceremonies of the East Indians, as Well Chyneses as Javans, Londres, Walter Burre, 1943 (orig. 1606), p. 173. Según Jean Chretien Baud, las primeras referencias al madak en las fuentes holandesas lo ubican en la isla de Ternate, en 1610. Jean C. Baud, "Proeve van eene Geschiedenis van den Handel en het Verbruik van Opium in Nederlandsch Indië", Bijdragen tot de Taal-, Land-en Volkenkunde van Nederlandsch-Indië, vol. 1, núm. 1, 1853, p. 80. 
nueva dimensión a su consumo, al pasar de ser ingerido a ser fumado en pipa, lo que transformó significativamente su apreciación social, al disociarlo de su función medicinal original. ${ }^{33}$

En paralelo a este proceso, durante la segunda mitad del siglo XVII, los holandeses en India comenzaron a adquirir y vender opio para obtener una ventaja comercial en las adquisiciones de pimienta, lo que los convirtió en intermediarios del comercio del opio bengalí en la costa malabar. ${ }^{34}$ Estas experiencias fueron trasladadas de forma extensiva a Java tras la conquista de Batavia en 1619, y generaron un tráfico en el que los agentes de la Vereenigde Oost-Indische se reservaron los derechos monopolísticos del suministro de la droga en dicho puerto a partir de $1659 .{ }^{35} \mathrm{El}$ resultado fue una tendencia de crecimiento del suministro, en un doble proceso de incremento de la oferta y aumento del precio del opio. El análisis realizado por Souza demuestra que, entre 1672 y 1717, tanto el precio del opio en Bengala como el del revendido en Yakarta crecieron más del triple, mientras que, por su parte, el montante de las exportaciones a Batavia se multiplicó por más de nueve veces. ${ }^{36} \mathrm{Si}$ bien estos datos dan cuenta del modo en el que los holandeses se beneficiaron de este tráfico, aquí nos interesa ver cómo éste demuestra la capacidad disruptiva de la droga en los patrones comerciales, que correspondería a la primera fase del modelo Bradburd-Jankowiak. Dado que el opio no consiguió ser cultivado en Java hasta las primeras décadas del siglo XIX, los holandeses se hicieron con el control de un comercio pre-

${ }^{33}$ Este proceso se observa de forma paralela en lugares como Persia. Rudi Matthee, The Pursuit of Pleasure: Drugs and Stimulants in Iranian History, 1500-1900, Nueva Jersey, Princeton University Press, 2005, pp. 207-236.

${ }^{34}$ Derk, History of the Opium Problem, op. cit., pp. 163-170.

${ }^{35}$ Esta práctica se vería complementada con la adquisición de derechos exclusivos para la venta de opio en lugares como Mataram y Ceribon, entre 1677 y 1678 . James Rush, Opium to Java: Revenue Farming and Chinese Enterprise in Colonial Indonesia, 1860-1910, Singapur, Equinox Publishing, 2007, p. 26.

${ }^{36} \mathrm{El}$ análisis de los archivos de la Vereenigde Oost-Indische Compagnie, realizado por Souza, muestra un crecimiento del opio bengalí enviado a Batavia de un promedio de 92 cajas entre 1667 y 1676 a las 853 de la década comprendida entre 1707 y 1716. Igualmente, saca a relucir una oscilación en su precio en Bengala de 60 a 170 rupias y de 200 a 572 rupias en Batavia, entre 1672 y 1717. George B. Souza, "Developing Habits: Opium and Tobacco in the Indonesian Archipelago, c. 1619-c. 1794”, en James H. Mill y Patricia Barton (eds.), Drugs and Empires: Essays in Modern Imperialism and Intoxication, c. 1500-c. 1930, Nueva York, Palgrave MacMillan, 2007, pp. 46-47. 
existente que provocaría su sustancial redefinición, y crearía un modelo que concentraba todo el abastecimiento en Batavia, para dejar la distribución por la costa norte de la isla a los comerciantes locales, especialmente los chinos. ${ }^{37}$ En su caso, el opio se convirtió en el artículo principal en sus actividades, sustituyó productos de lujo como el marfil, los nidos de pájaros o los pepinos de mar, y provocó a su vez la difusión de su consumo a Malasia. ${ }^{38}$

Volvamos a China. Las actividades holandesas en el Mar del Sur de China se han expuesto como el principal factor que causó la entrada de esta nueva forma de consumo en el ámbito chino. Owen argumenta que este proceso vino provocado por la ocupación holandesa de las regiones meridionales de la costa de Taiwan, entre 1624 y 1662, tras lo cual el hábito quedó entre los isleños; pasó después a la China continental luego de su conquista por las fuerzas Qing, en 1683, factor que ha sido además valorado por Zheng Yangwen, al presentarlo como la principal vía para la subsecuente apreciación del opio entre la élite china del momento. ${ }^{39}$ No obstante, la visión que intentamos plantear critica este enfoque, en tanto que no toma en consideración ciertos aspectos técnicos como el refinamiento del opio previo a la producción del madak, ${ }^{40}$ y sobre todo por la poca importancia de la isla dentro de las redes comerciales entre China y el Sureste Asiático.

Vistas desde una óptica más general, las primeras etapas del comercio del opio llevado a cabo por los europeos en el siglo XVIII se dieron en un periodo marcado por el florecimiento de la actividad comercial china en el Sureste Asiático, que se hizo notar no sólo en las costas del mar de China meridional, sino también en Vietnam y Tailandia, Estados cuya forma-

${ }^{37}$ Lucas W. Nagtegaal, Riding the Dutch Tiger: The Dutch East Indies Company and the Northeast Coast of Java, 1680-1743, Leiden, KITLV Press, 1996, pp. 143-147.

${ }^{38}$ Kwee Hui Kian, The Political Economy of Java's Northeast Coast c. 1740-1800, Boston-Leiden, Brill, 2006, pp. 24-25; Zheng, The Social Life of Opium in China, op. cit., pp. 41-44.

${ }^{39}$ Zheng, The Social Life of Opium in China, op. cit., pp. 45-48; Owen, British Opium Policy in China and India, op. cit., pp. 15-16.

${ }^{40}$ Recientemente, este aspecto ha levantado cierto escepticismo en autores como Carl A. Trocki ("Opium as a Commodity in the Chinese Nanyang Trade”, en Eric Tagliacozzo y Wen-Chin Chang [eds.], Chinese Circulations: Capital, Commodities, and Networks in Southeast Asia, Durham, Duke University Press, 2011, p. 102, nota 6). 
ción deriva del crecimiento económico y poblacional acaecidos durante dicho periodo. ${ }^{41}$ En lo que a China se refiere, durante la primera mitad del siglo XVIII (1724-1750), puertos como Cantón o Fuzhou experimentaron un incremento de la entrada de productos de estas regiones, impulsado por una creciente demanda causada por la explosión demográfica que caracterizó el reinado del emperador Qianlong (1735-1795). ${ }^{42}$ En este sentido, las actividades de holandeses y británicos no fueron ajenas al papel del opio en el renacer de la actividad comercial en la región, ya que constituía una de sus mercancías más atractivas y rentables. ${ }^{43}$ En lo que respecta a los segundos, esto se hace perceptible en un contexto en el que, por coincidir con la fundación del reino de Siam, en 1782, y de su capital, Bangkok, surgió un nuevo organigrama de articulación de este renacer de la actividad comercial, del que formarían parte diversos núcleos a los que posteriormente se adheriría Singapur. La presencia de esta droga se constata en los ejes comerciales que conectaron a Bangkok con la Batavia holandesa durante la segunda mitad del siglo XVIII, así como con la Penang británica y Singapur durante las primeras décadas del siglo XIX. Igualmente, ha quedado constancia de ella en la línea comercial que unió a Vietnam con Singapur durante las décadas de 1830 y $1840 .{ }^{44}$

Desde esta perspectiva se comprende que, en esencia, el comercio del opio y su crecimiento a lo largo de los siglos XVIII y XIX no fueron fruto de dinámicas provocadas por los británicos; pero sí es cierto que, a diferencia de otros actores europeos, ellos fueron los primeros en materializar el sueño de Albuquerque al controlar a través de la East India Company

${ }^{41}$ Victor Lieberman, "Local Integration and Eurasian Analogies: Structuring Southeast Asian History, c. 1350-1830”, Modern Asian Studies, vol. 27, núm. 3, 1993, pp. 475-572.

${ }^{42} \mathrm{Li}$ Tana, “The Water Frontier”, en Nola Cooke y Li Tana (eds.), Water Frontier: Commerce and the Chinese in the Lower Mekong Region, 1750-1880, Singapur, Singapore University Press, 2004, pp. 1-17.

${ }^{43}$ Recientes estudios dan cuenta de la vital importancia del comercio del opio en el sostenimiento de la presencia de la Vereenigde Oost-Indische Compagnie en lugares como Java. George B. Souza, "Opium and the Company: Maritime Trade and Imperial Finances on Java, 1684-1796”, Modern Asian Studies, vol. 43, núm. 1, 2009, pp. 113-133.

${ }^{44}$ Anthony Reid, "Chinese Trade and Southeast Asian Economic Expansion in the Later Eighteenth and Early Nineteenth Centuries: An Overview”, en Cooke y Li (eds.), Water Frontier, op. cit., pp. 31-32. 
la producción de opio de las provincias de Bengala, Bihar y Uttar Pradesh, principales abastecedoras del mercado asiático de opio en el momento. ${ }^{45}$ Este cambio vino ligado a una conflictiva redefinición de los criterios de producción, trabajo y organización territorial que tomaron cuerpo con la creación del sistema de contrataciones, ideado por el marqués Charles de Cornwallis (1738-1805), que fue empleado desde 1797 y que adquirió carta de naturaleza jurídica en $1799 .{ }^{46} \mathrm{El}$ opio no jugó ciertamente un papel trascendental en la colonización de India si atendemos a su consumo, pero sí intervino en el desarrollo de ciertas técnicas de explotación, e incluso apropiación, de la fuerza de trabajo por el deseo de controlar la producción; ${ }^{47}$ además, en lo que a su papel en el ascenso colonial británico en Asia se refiere, cabe resaltar que la creación de este monopolio permitió el abaratamiento sustancial de la obtención de la droga, la cual era después vendida en Calcuta en pública subasta.

Por otra parte, este nuevo mercado de opio desempeñó una función relevante en la consolidación definitiva de los country traders, comerciantes privados, frente a las compañías monopolísticas, como vía de articulación de la presencia colonial en Asia. En esta línea, es necesario puntualizar que el comercio privado fuera del control de monopolios o compañías comerciales había sido ya una característica esencial de la actividad mercantil europea en Asia desde el siglo Xvi; surgió como respuesta a la necesidad de los europeos de adaptarse a un entorno comercial marcado por la estacionalidad impuesta por los monzones, lo que hacía necesaria su participación en los circuitos locales a

${ }^{45}$ No fue sino hasta el siglo XIX cuando el opio de otras regiones adquirió más importancia en este contexto, especialmente el turco. Jacques M. Downs, "American Merchants and the China Opium Trade, 1800-1840", The Business History Review, vol. 42, núm. 4, 1968, pp. 418-442.

${ }^{46}$ Trocki, Opium, Empire and the Global Political Economy, op. cit., pp. 61-66; Narayan Prasad Singh, The East India Company's Monopoly Industries in Bihar with Particular Reference to Opium and Saltpetre, 1773-1833, Muzaffarpur, Sarvodaya Vangmaya, 1980; John F. Richards, "The Indian Empire and Peasant Production of Opium in the Nineteenth Century", Modern Asian Studies, vol. 15, núm. 1, 1981, pp. 59-82, y Om Prakash, "Opium Monopoly in India and Indonesia in the Eighteenth Century", Indian Economic Social History Review, vol. 24, núm. 1, 1987, pp. 63-80.

${ }^{47}$ Esto quedó reflejado principalmente en el periodo que comprende los primeros 20 años de la instauración del monopolio (1773-1793), marcado por los abusos de los contratistas ingleses sobre los agricultores indios en las regiones adscritas a la producción de opio. Richards, “The Indian Empire...”, op. cit., pp. 63-65. 
fin de poder rentabilizar la inversión de tiempo que suponía esperar que los vientos fuesen favorables. Sin embargo, esta fórmula no fue apoyada abiertamente por ningún Estado hasta que la situación de la prohibición del comercio y el consumo de opio en China provocó que el contrabando se convirtiera en el modo en que la East India Company podía colocar la droga en China, cumpliendo así una doble función, como era desvincular el comercio de la droga de la Compañía y colocar la plata obtenida de su venta en Cantón, donde le era más necesaria para la compra de té. ${ }^{48}$ Así, el imparable desarrollo de este nuevo comercio del opio estaría detrás de la formación de grandes empresas privadas británicas en Asia, como la Jardine, Matheson \& Co. ${ }^{49}$ y supuso una de las causas esenciales de la definitiva disolución del monopolio en 1833, en tanto que este comercio privado comenzó a abastecerse de opio de Malwa, región no controlada por el monopolio, frente al de Calcuta. ${ }^{50} \mathrm{En}$ esta línea, la historiografía más reciente ha demostrado sobradamente la dependencia económica del entonces gobierno de India del comercio del opio con China llegado el ecuador del siglo XIX, ya que era una pieza clave de la financiación no sólo del dominio británico en el subcontinente, sino también de su presencia en toda Asia. ${ }^{51}$

\section{Conclusiones}

A modo de conclusión planteo que el comercio del opio llevado a cabo por los europeos en China y su área de influencia mercatil jugó un papel relevante en la despolarización del sistema productivo sinocentrado en Asia oriental, central y suroriental, que dominó la época precolonial. Al aplicar el

${ }^{48}$ Trocki, Opium, Empire and the Global Political Economy, op. cit., pp. 48-52.

${ }^{49}$ Robert Blake, Jardine Matheson: Traders of the Far East, Londres, Weidenfeld \& Nicolson, 1999, pp. 39-41.

${ }_{50}$ Amar Farooqui, Smuggling as Subversion: Colonialism, Indian Merchants and the Politics of Opium, Nueva Delhi, New Age International Publisher, 1998, pp. 13-15.

${ }^{51}$ Quizás el análisis más completo de las implicaciones del comercio del opio entre India y China en la economía del Imperio británico se encuentre en Wong, Deadly Dreams, op. cit., pp. 333-454. 
modelo de Hamashita de relaciones centro-periferia al comercio del opio es posible vislumbrar un nuevo marco teórico, donde la utilización del opio fue sustancial en su dislocación y en la penetración colonial europea en Asia durante los siglos XVIII y XIX; para ello, es imprescindible recurrir a los recientes aportes de la antropología -como el de Bradburd y Jankowiak-que han sistematizado el uso de las drogas en el proceso colonial con una primera fase centrada en el control comercial. Como he analizado, el caso de estudio se ajusta completamente a este modelo teórico, ya que tanto en las actividades de los holandeses en Indonesia y el Sureste Asiático como, posteriormente, en las operaciones de los británicos en China, el comercio de este artículo fue esencial no sólo en la financiación de la empresa colonial de ambos imperios, sino también especialmente en la transformación de las relaciones comerciales en las regiones involucradas. Sólo un producto como el opio, con su capacidad inherente para transformar los patrones de consumo y trastocar los principios básicos de intercambiabilidad, plasmada en el aumento incontrolado de su demanda y su precio, pudo dar a estos actores la ventaja cualitativa en un entorno que no eran capaces de controlar.

Así, la mercantilización del opio en un sentido moderno comenzó incluso antes de que los británicos se hiciesen con el monopolio del opio indio en 1773, y contribuyó a la penetración holandesa en la periferia del sistema comercial sinocentrado imperante. No obstante, fue en los últimos compases del siglo XVIII cuando entró en sus últimas fases, y trajo consigo el desarrollo definitivo del poder de esta droga para transformar sociedades y economías, a lo que se asocia la necesidad de su control por los poderes políticos en la región hasta bien entrado el siglo xx. ${ }^{52}$ A este proceso se sumaría posteriormente la legalización del comercio del opio en China, refrendada en 1858, con el Tratado de Tianjin, lo cual debe leerse como una demostración de fuerza de los poderes imperialistas occidentales en un doble sentido, ya que no sólo autorizaba la injerencia del capital europeo en la economía china, sino que además permitía la imposición de una determinada política de consumo

${ }^{52}$ Brook y Wakabayashi (eds.), Opium Regimes, op. cit., pp. 1-5. 
que suponía, en definitiva, arrebatar a la administración Qing su soberanía en política económica.

Dirección institucional del autor:

Departamento de Lingüistica y Teoría de la Literatura

Facultad de Filosofía y Letras

Universidad de Granada, Campus de Cartuja

18007 Granada, España

jacantonal@gmail.com/canton@correo.ugr.es

\section{Bibliografía}

Appadurai, Arjun (ed.), The Social Life of Things: Commodities in Cultural Perspective, Cambridge, Cambridge University Press, 1986.

ARRIGHI, Giovanni, Chaos and Governance in the Modern World System, Minneapolis, University of Minnesota Press, 1999.

Arrighi, Giovanni, Iftikhar Ahmad y Miin-wen Shih, "Western Hegemonies in World Historical Perspective", en Giovanni Arrighi, Chaos and Governance in the Modern World System, Minneapolis, University of Minnesota Press, 1999, pp. 227236.

BARBOSA, Duarte, Livro em que se dá relação do que viu e oviu no oriente Duarte Barbosa, Lisboa, Divisão de publicações e biblioteca agência geral das colònias, 1956.

BAUD, Jean C., "Proeve van eene Geschiedenis van den Handel en het Verbruik van Opium in Nederlandsch Indië", Bijdragen tot de Taal-, Land-en Volkenkunde van Nederlandsch-Indië, vol. 1, núm. 1, 1853, pp. 79-220.

Baumler, Alan (ed.), Modern China and Opium: A Reader, Ann Arbor, University of Michigan Press, 2001.

Blake, Robert, Jardine Matheson: Traders of the Far East, Londres, Weidenfeld \& Nicolson, 1999.

BRADBURD, Daniel y William Jankowiak (eds.), Drugs, Labor and Colonial Expansion, Tucson, The University of Arizona Press, 2003. Brook, Timothy y Bob T. Wakabayashi (eds.), Opium Regimes. China, Britain and Japan, 1839-1952, Berkeley-Los Ángeles, University of California Press, 2000.

Chopra, R. N., Indigenous Drugs of India, Calcuta, The Art Press, 1933. 
Cooke, Nola y Li Tana (eds.), Water Frontier: Commerce and the Chinese in the Lower Mekong Region, 1750-1880, Singapur, Singapore University Press, 2004.

Das GuPTA, Ashin y Michael N. Pearson, India and the Indian Ocean, 1500-1800, Oxford, Oxford University Press, 1987.

De Bulhão Pato, Raymondo A. (ed.), Cartas de Affonso de Albuquerque, seguidas de documentos que as elucidam, t. I, Lisboa, Typographia da Academia Real das Sciencias, 1884.

De Orta, Garcia, Colóquios dos simples e drogas da India, vol. 2, Lisboa, Academia Real das Sciencias de Lisboa, 1891.

Derk, Hans, History of the Opium Problem: The Assault on the East, ca. 1600-1950, Leiden, Brill, 2012.

DiköTTER, Frank, Lars Laamann y Zhou Xun, Narcotic Culture: A History of Drugs in China, Chicago, University of Chicago Press, 2004.

Downs, Jacques M., “American Merchants and the China Opium Trade, 1800-1840”, The Business History Review, vol. 42, núm. 4, 1968, pp. 418-442.

Escohotado, Antonio, Historia general de las drogas, Madrid, Espasa, 2005.

Faibank, John K., Trade and Diplomacy on the China Coast: The Opening of the Treaty Ports, 1842-1854, Cambridge, Harvard University Press, 1953.

FAROoQui, Amar, Smuggling as Subversion: Colonialism, Indian Merchants and the Politics of Opium, Nueva Delhi, New Age International Publisher, 1998.

Foxcroft, Louise, The Making of Addiction: The "Use and Abuse" of Opium in Nineteenth-Century, Aldershot, Ashgate Publishing, 2013.

Frank, Andre G., ReOrient: Global Economy in the Asian Age, Berkeley-Los Ángeles, University of California Press, 1998.

Gang, Zhao, The Qing Opening to the Ocean: Chinese Maritime Policies, 1684-1757, Honolulú, Hawaii University Press, 2013.

Gilman, Sander L. y Zhou Xun (eds.), Smoke: A Global History of Smoking, Londres, Reaktion Books, 2004.

GONG, Yingyan 龚姃晏, Yapian de chuanbo yu duibua yapian maoyi 鸦片的传播与对华鸦片贸易, Beijing, Dongfang Chubanshe 东 方出版社, 1999.

Goodman, Jordan, Tobacco in History: The Cultures of Dependence, Londres, Routledge, 2005.

Hamashita, Takeshi, China, East Asia and the Global Economy: Regional and Historical Perspectives, Oxon, Routledge, 2008. 
HunT, Geoffrey y Judith C. Barker, "Socio-cultural Anthropology and Alcohol and Drug Research: Towards a Unified Theory", Social Science E Medicine, vol. 53, núm. 2, 2001, pp. 165-188.

Kian, Kwee Hui, "Pockets of Empire: Integrating the Studies on Social Organizations in Southeast China and Southeast Asia", Comparative Studies of South Asia, Africa and the Middle East, vol. 27, núm. 3, 2007, pp. 616-632.

Kinn, Kwee Hui, The Political Economy of Java's Northeast Coast c. 1740-1800, Boston-Leiden, Brill, 2006.

Klein, Peter, "The China Seas and the World Economy between the Sixteenth and Nineteenth Centuries: The Changing Structure of World Trade", en Carl-Ludwig Holtfrerich (ed.), Interaction in the World Economy. Perspectives from International Economic History, Londres, Harvester, 1989, pp. 61-89.

Li, Tana, "The Water Frontier”, en Nola Cooke y Li Tana (eds.), Water Frontier: Commerce and the Chinese in the Lower Mekong Region, 1750-1880, Singapur, Singapore University Press, 2004, pp. 1-17.

LiEBERMAN, Victor, "Local Integration and Eurasian Analogies: Structuring Southeast Asian History, c. 1350-1830", Modern Asian Studies, vol. 27, núm. 3, 1993, pp. 475-572.

MatTern, Susan P., The Prince of Medicine: Galen in the Roman Empire, Oxford, Oxford University Press, 2013.

Matthee, Rudi, The Pursuit of Pleasure: Drugs and Stimulants in Iranian History, 1500-1900, Nueva Jersey, Princeton University Press, 2005.

MerLin, Mark D., On the Trail of the Ancient Opium Poppy, Nueva Jersey, Fairleigh Dickinson University Press, 1984.

MilleR, William R. y Kathleen M. Carroll, Retbinking Substance Abuse: What the Science Shores, and What We Should Do about It, Nueva York, Guilford Press, 2006.

Moore, Jason W., "Madeira, Sugar, and the Conquest of Nature in the 'First' Sixteenth Century, Part I: From 'Island of Timber' to Sugar Revolution, 1420-1506", Review: A Journal of the Fernand Braudel Center, vol. xxxII, núm. 4, 2009, pp. 345-390.

Moore, Jason W., "Madeira, Sugar, and the Conquest of Nature in the 'First' Sixteenth Century, Part II: From Local Crisis to Commodity Frontier, 1506-1530", Review: A Journal of the Fernand Braudel Center, vol. XxxIII, núm. 1, 2010, pp. 1-24.

NagtegaAl, Lucas W., Riding the Dutch Tiger: The Dutch East Indies Company and the Northeast Coast of Java, 1680-1743, Leiden, KITLV Press, 1996. 
Owen, David E., British Opium Policy in China and India, New Haven, Yale University Press, 1934.

PraKash, Om, "Opium Monopoly in India and Indonesia in the Eighteenth Century", Indian Economic Social History Review, vol. 24, núm. 1, 1987, pp. 63-80.

ReID, Anthony, "Chinese Trade and Southeast Asian Economic Expansion in the Later Eighteenth and Early Nineteenth Centuries: An Overview", en Nola Cooke y Li Tana (eds.), Water Frontier: Commerce and the Chinese in the Lower Mekong Region, 1750-1880, Singapur, Singapore University Press, 2004, pp. 21-34.

RicHARDS, John F., "The Indian Empire and Peasant Production of Opium in the Nineteenth Century", Modern Asian Studies, vol. 15, núm. 1, 1981, pp. 59-82.

Ricklefs, M., "The Crisis of 1740-1 in Java: The Javanese, Chinese, Madurese and Dutch, and the Fall of the Court of Kartasura", Bijdragen tot de Taal-, Land-en Volkenkunde, vol. 139, núm. 2, 1983, pp. 268-290.

Rush, James, Opium to Java: Revenue Farming and Chinese Enterprise in Colonial Indonesia, 1860-1910, Singapur, Equinox Publishing, 2007.

ScArborough, John, "The Opium Poppy in Hellenistic and Roman Medicine”, en Roy Porter (ed.), Drugs and Narcotics in History, Cambridge, Cambridge University Press, 1997, pp. 4-23.

Schottenhammer, Angela, "Huihui Medicine and Medicinal Drugs in Yuan China", en Morris Rossabi (ed.), Eurasian Influences on Yuan China, Singapur, Institute of Southeast Asian Studies, 2013. Scotт, Edmund, An Exact Discourse of the Subtilties, Fashishions [sic], Pollicies, Religion and Ceremonies of the East Indians, as Well Chyneses as Javans, Londres, Walter Burre, 1943.

SherratT, Andrew, "Sacred and Profane Substances: The Ritual Use of Narcotics in Later Neolithic Europe", en P. Garwood, D. Jennings, R. Skeates y J. Toms (eds.), Sacred and Profane: Proceedings of a Conference on Archaeology, Ritual and Religion, Oxford, University Committee for Archaeology Monographs, 1991, pp. 50-64.

SINGH, Narayan Prasad, The East India Company's Monopoly Industries in Bihar with Particular Reference to Opium and Saltpetre, 17731833, Muzaffarpur, Sarvodaya Vangmaya, 1980.

SouzA, George B., "Developing Habits: Opium and Tobacco in the Indonesian Archipelago, c. 1619-c. 1794”, en James H. Mill y Patricia Barton (eds.), Drugs and Empires: Essays in Modern Im- 
perialism and Intoxication, c. 1500-c. 1930, Nueva York, Palgrave MacMillan, 2007, pp. 39-56.

SouzA, George B., "Opium and the Company: Maritime Trade and Imperial Finances on Java, 1684-1796”, Modern Asian Studies, vol. 43, núm. 1, 2009, pp. 113-133.

SteEnsgaARD, Niels, "The Companies as a Specific Institution in the History of European Expansion”, en L. Blussé y F. Gaastra (eds.), Companies and Trade: Essays on Overseas Trading Companies during the Ancient Regime, Leiden, Leiden University Press, 1981, pp. 245-264.

STEENSGAaRD, Niels, "The Dutch East India Company as an Institutional Innovation", en M. Aymard (ed.), Dutch Capitalism and World Capitalism, Cambridge, Cambridge University Press, 1982, pp. 235-257.

Subrahmanyam, Sanjay, The Political Economy of Commerce. Southern India, Cambridge, Cambridge University Press, 1990.

Tejapira, Kasian, "Pigtail: A Prehistory of Chineseness in Siam", en Tong Chee Kiong y Chan Kwok B. (eds.), Alternate Identities: The Chinese of Contemporary Thailand, Leiden, Brill, 2001.

TILBI, Selma, The Medicinal Use of Opium in Ninth-Century Baghdad, Leiden, Brill, 2006.

Trocki, Carl A., "Opium as a Commodity in the Chinese Nanyang Trade”, en Eric Tagliacozzo y Wen-Chin Chang (eds.), Chinese Circulations: Capital, Commodities, and Networks in Southeast Asia, Durham, Duke University Press, 2011, pp. 84-104.

Trocki, Carl A., Opium, Empire and the Global Political Economy, Londres, Routledge, 1999.

Van Dyke, Paul A., Merchants of Canton and Macao: Politics and Strategies in Eighteenth-Century Chinese Trade, Hong Kong, Hong Kong University Press, 2011.

Van Dyke, Paul A., The Canton Trade: Life and Enterprise on the China Coast, 1700-1845, Hong Kong, Hong Kong University Press, 2005.

Wong, John Y., Deadly Dreams: Opium and the Arrow War (18561860) in China, Cambridge, Cambridge University Press, 1998. Zheng Yangwen, The Social Life of Opium in China, Cambridge, Cambridge University Press, 2005.

ZHONG Weimin 仲伟民, Chaye yu yapian: shijiu shiji quanqiubua zhong de Zhongguo 茶叶与鸦片: 十九世纪经济全球化中的中 国, Beijing, Shenghuo, Dushu, Xinzhi Sanlian Shudian 生活・ 读书・新知三联书店, 2010. 